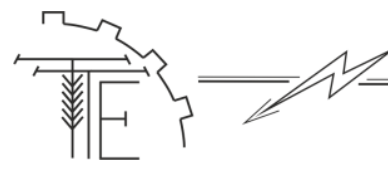

Vol. 115, No 4 / 2021

UDC 621.43.001.42

DOI: $10.37128 / 2520-6168-2021-4-3$

\title{
REMOVAL OF TECHNICAL AND ECONOMIC INDICATORS OF THE D-240 ENGINE WHEN USING BIOFUELS BY APPLYING THE DIESEL-RK SOFTWARE COMPLEX
}

\author{
Serhii Burlaka, Ph.D., Senior Lecturer \\ Tokarchuk Oleksii, Ph.D., Associate Professor \\ Vinnytsia National Agrarian University
}
Бурлака Сергій Андрійович, доктор філософії, старший викладач
Токарчук Олексій Анатолійович, к.т.н., доцент
Вінницький національний аграрний університет

\begin{abstract}
Ukraine is one of the energy-deficient countries because it covers its needs in fuel and energy resources from domestic reserves by only 53\% (imports $75 \%$ of the required natural gas and $85 \%$ of crude oil and petroleum products). Dependence on oil imports is seen by most developed countries as a matter of national and energy security, and the use of petroleum products as energy sources poses a significant environmental risk.

According to the analysis of the state of the world engine industry, the most effective measures to improve the design of the engine are: development and implementation of a new workflow with effective methods of mixing and combustion; development and creation of the design of the system of automatic regulation of diesel rotation. frequency to improve fuel economy And to reduce toxicity in partial load mode, some cylinders are switched off at idle.

To strengthen the requirements for fuel consumption standards and the level of toxicity of exhaust gases, as well as to increase the reliability and efficiency of agricultural power engines, it is necessary to formulate measures to improve its design.

The choice of a fuel should be determined by the optimal combination of environmental and economic performance of the engine. Prospects for the use of a particular fuel of plant origin are noted. If for fuels of petroleum origin the improvement of its properties is determined by special processing, then oils with the set characteristics can be received already in "field" by selection of the corresponding grades of plants, use of fertilizers, agronomic actions, etc.
\end{abstract}

The problem of reducing the consumption of diesel fuel at idle and low load can be solved by excluding from its operation part of the cylinders (this method is widely used) and closing the cycle of one cylinder.

A comparative analysis of the main technical and environmental performance of the D-240 diesel engine when working on traditional and alternative fuels using the computer program Diesel-RK.

Keywords: diagnostics, engine, indicators, biofuel, D-240, indicator indicators, technical and economic characteristics

F. 2. Fig. 9. Table 4. Ref. 9.

\section{Formulation of the problem}

The development and commissioning of new fuels requires theoretical and experimental research to be able to record the status and environmental performance of a diesel engine when using different fuels.

Experience of practical use of liquid biofuels, such as rapeseed oil (RA), in internal combustion engines (ICE) shows that the design of ICE does not provide for the use of this type of hydrocarbon fuel, as biofuels are not designed for this type of fuel combustion plant, and its use causes a number serious operational problems, such as loss of engine power; inability to start the engine from a cold state; failure of exhaust valves; reduction of service life of the fuel equipment (fuel pumps (PN) and atomizer sprays); increase of injection pressure to 25\%; violation of the conditions of the organization of the fuel combustion process in the combustion chamber [1].

Biofuels are aggressive against certain sealing materials, in particular rubber products, paints and varnishes, as well as certain non-ferrous metals (aluminum, zinc, copper and their alloys). In most cases, this 


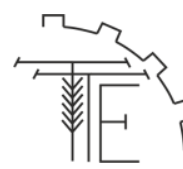

phenomenon is caused by imperfection or simplification of the technological process of production of esters (insufficient removal of catalyst, methanol residues, lack of neutralization of oil, etc.).

In terms of heat of combustion, biofuel is inferior to mineral diesel fuel $(37.2 \mathrm{MJ} / \mathrm{kg}$ in biofuels against $42.5 \mathrm{MJ} / \mathrm{kg}$ in mineral diesel fuel). Therefore, the power of a biofuel engine is reduced by an average of $7 \%$, and fuel consumption increases by about 5-8\%. In addition, biofuels have less resistance to oxidation compared to diesel fuel, which is especially important for long-term storage of ethers in pure form. Oxidation can lead to an increase in acid number and viscosity, as well as the formation of harmful compounds (resins) that can block fuel filters. Excessive acidity may be due to inconsistent quality characteristics of raw materials. Therefore, esters should not be stored for more than 6 months, otherwise antioxidants should be added to them [2].

The identified operational problems are tried to be solved by giving the biofuel a set of properties that correspond to the properties of traditional petroleum fuel, adding to its composition various additives and additives, such as alcohols (methyl, ethyl) and esters (methyl-tert-butyl).

Another way to solve problems with the use of biofuels, namely operational, is to change the design of heat engines and fuel plants. Design and development of internal combustion engines, gas turbines should be carried out for a specific type of biofuel, such as rapeseed, colza, burdock or flax. The implementation of measures in this area has prospects, but their practical implementation requires a long time and significant financial investment [3].

Another area is the combustion of biofuels as part of fuel hydrocarbon mixtures, which include as the main components $50-70 \%$ of petroleum fuels and 30-50\% of biofuels; adding to biofuel properties of molecular structure and structure similar to regular petroleum fuel (jet-cavitation and (or) rotor-pulsation processing).

To calculate the performance and determine the impact of biofuels and their mixtures on the D-240 engine, the program "Diesel-RK" is used.

The D-240 engine (4Ch11/12,5) is installed on the MTZ-80 general-purpose tractor (82) and selfpropelled agricultural machinery [4].

\section{Analysis of recent research and publications}

All types of promising alternative fuels currently used in internal combustion engines, by type of raw material can be divided into large groups: petroleum, biomass and even gases of various origins [5].

To date, there are many technologies for producing diesel fuel from biomass as a renewable raw material.

This issue was addressed by: G.M. Kaletnik, M.G. Sandomirsky, L.P. Wednesday, W.F. Anisimov, Ya.Yu. White and many others.

Recently, scientific research in the field of rapeseed oil and its products - methyl or ethyl esters - has become widespread. For example, in Germany, a tractor diesel "Steur WD 408/43" was tested on a mixture of $\mathrm{RO}$ and diesel fuel in equal proportions. After 287 hours of operation, there was ringing of the rings, tarring of the exhaust channel and a large deposit on the exhaust valves, which means that part of the oil did not participate in the combustion process, and getting on the parts of the cylinder-piston group, formed scale [6].

\section{The aim of the study}

Carry out computer simulation of the D-240 engine using biofuels and its mixtures by using the DIESEL-RK software package.

\section{Presenting main material}

According to research and analysis in the country and abroad, the transition of diesel engines with a traditional design scheme to alternative fuels, especially biofuels based on vegetable oil, has caused a certain degree of deterioration of their normal operation. Nozzle holes and their coking. When the engine is running on oil instead of fuel for a long time, a large amount of soot in the combustion chamber can cause the engine to malfunction [7].

The calculation program provides for the determination of technical and environmental indicators during engine operation at the crankshaft speed $n=2200 \mathrm{~min}^{-1}$ (rated power mode) and the maximum torque mode ( $\mathrm{n}=1600$ $\min ^{-1}, \mu_{\mathrm{r}}=298 \mathrm{~N} \cdot \mathrm{m}$ ). The type of fuel is determined: diesel fuel (DP) $-100 \%$, biofuel B100 and mixtures B5 and B20.

Some calculation dependencies used by the software package are given below.

Residual gas ratio $[8,9]$ : 


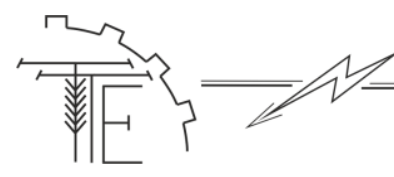

Vol. 115, No 4 / 2021

$$
\gamma_{r}=\frac{P_{r}\left(T_{O}+\Delta T\right)}{\left(\varepsilon \cdot P_{a}-P_{r}\right) \cdot T_{r}}
$$

where $\mathrm{T}_{\mathrm{O}}=288^{\circ} \mathrm{K}$ - ambient air temperature, $\varepsilon=16,0$ - the degree of compression according to the technical characteristics of the engine, $P_{r}=0,108 \mathrm{MPa}$ - exhaust gas pressure, $\operatorname{Tr}=750^{\circ} \mathrm{K}$ - the temperature of the exhaust gases, $\mathrm{P}_{0}=0,1 \mathrm{MPa}$ - atmospheric pressure, $\mathrm{P}_{\mathrm{a}}$ - pressure at the end of the intake stroke, $\mathrm{MPa}, \Delta \mathrm{T}=20{ }^{\circ} \mathrm{K}$ - heating temperature of the charge at the inlet.

$$
\mathrm{P}_{\mathrm{a}}=\cdot \mathrm{P}_{0}-\Delta \mathrm{P} \text {. }
$$

The pressure loss at the inlet $\Delta \mathrm{P}_{\mathrm{a}}$ is calculated by the formula:

$$
\Delta P_{a}=\left(\beta^{2}+\xi_{B \Pi}\right) \cdot \frac{\omega^{2}{ }_{B \Pi}}{2} \cdot \rho_{o} \cdot 10^{-6}, \mathrm{MPa}
$$

where $\beta$-the damping coefficient of the charge velocity in the considered cross section of the cylinder, $\xi_{B \Pi}$-the coefficient of resistance of the intake system, $\omega_{B \Pi}-$ the average speed of the charge in the smallest cross section of the intake system [9].

The calculated dependences of the D-240 engine when working on different types of fuel modeled in the software package DIESEL-RK are given in table. 1-4.

It is also worth paying attention to the comparative physico-chemical composition of diesel fuel (biofuel), which determines the specifics of the diesel engine D-240 when using biofuel, which is presented in Fig. 1.

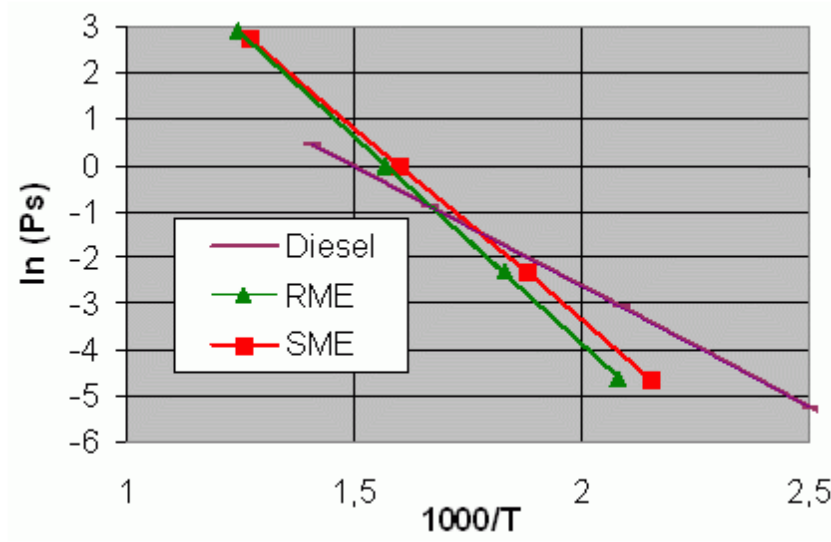

Fig. 1. Couple vapor pressure of pure fuels depending on temperature

Table 1.

\section{Effective performance of D-240 when working on Biofuel SME B5}

--- PARAMETERS OF EFFICIENCY AND POWER ---

2200.0 - RPM - Engine Speed, rev/min

56.990 - P_eng - Piston Engine Power, kW

6.5420 - BMEP - Brake Mean Effective Pressure, bar

247.39 - Torque - Brake Torque, $\mathrm{N} \mathrm{m}$

0.05468 - m_f - Mass of Fuel Supplied per cycle, $g$

0.25328 - SFC - Specific Fuel Consumption, kg/kWh

0.23834 - SFC_ISO - Specific Fuel Consumption in ISO, $\mathrm{kg} / \mathrm{kWh}$

0.35632 - Eta_f - Efficiency of piston engine

8.4212 - IMEP - Indicated Mean Effective Pressure, bar

0.45867 - Eta_i - Indicated Efficiency

9.1667 - Sp - Mean Piston Speed, m/s

1.5798 - FMEP - Friction Mean Effective Pressure, bar (Intern.Exp)

0.80549 - Eta_m - Mechanical Efficiency of Piston Engine

\section{ENVIRONMENTAL PARAMETERS}

1.0000 - po_amb - Total Ambient Pressure, bar

288.00 - To_amb - Total Ambient Temperature, K

1.0000 - p_Te - Exhaust Back Pressure, bar (after turbine)
1859.4 - T_max - Maximum Cylinder Temperature, $\mathrm{K}$ 6.0000-CA_p.max - Angle of Max. Cylinder Pressure, deg. A.TDC 19.000-CA_tmax-Angle of Max. CylinderTemperature, deg. A.TDC 3.3170 - dp/dTheta- Max. Rate of Pressure Rise, bar/deg. 1.8829 - Ring_Intn- Ringing / Knock Intensity, $\mathrm{MW} / \mathrm{m}^{2}$ 7645.9 - F_max - Max. Gas Force acting on the piston, $\mathrm{kg}$ System: Custom Fuel Injection System 728.76 - p_inj.max-Max. Sac Injection Pres. (before nozzles), bar 511.59 - p_inj.avr- Mean Sac Press. for Total Fuel Portion, bar 19.022 - d_32 - Sauter Mean Diameter of Drops, microns 20.000 - SOI - Start Of Injection or Ignition Timing, deg. B.TDC 30.474 - Phi_inj - Duration of Injection, CA deg. 10.708 - Phi_ign - Ignition Delay Period, deg.

- ... - calculated by modified Tolstov method : 10.7 9.2921 - SOC - Start of Combustion, deg. B.TDC 0.12256 - x_e.id - Fuel Mass Fraction Evaporated during Ignit. Delay 85.000 - Phi_z - Combustion duration, deg.

Phi_z 5\%= 4.2; Phi_z 50\%= 18.6; Phi_z 95\%=47.4 3.3546-Rs_tdc-Swirl Ratio in the Combustion Chamber at TDC 
0.98000 - po_afltr - Total Pressure after Induction Air Filter, bar ---- TURBOCHARGING AND GAS EXCHANGE --.-

0.98000 - p_C - Pressure before Inlet Manifold, bar

288.00 - T_C - Temperature before Inlet Manifold, K 0.09480 - m_air - Total Mass Airflow (+EGR) of Piston Engine, kg/s 0.0000 -Eta_TC - Turbocharger Efficiency

1.0554 - po_T - Average Total Turbine Inlet Pressure, bar

762.89 - To_T - Average Total Turbine Inlet Temperature, K

0.09727 - m_gas - Mass Exhaust Gasflow of Pison Engine, kg/s 1.7219 - A/F_eq.t - Total Air Fuel Equivalence Ratio (Lambda) 0.58077 - F/A_eq.t - Total Fuel Air Equivalence Ratio -0.29939 - PMEP - Pumping Mean Effective Pressure, bar 0.93294 -Eta_v - Volumetric Efficiency

0.91428 -Eta_vo - Volumetric Efficiency defined by Ambient Parameters 0.03602 - x_r - Residual Gas Mass Fraction

0.98413 - Phi - Coeff. of Scavenging (Delivery Ratio / Eta_v) 0.53920 - BF_int - Burnt Gas Fraction Backflowed into the Intake, \% 1.5682 - \%Blow-by - \% of Blow-by through piston rings

0.97136 -p_int - Average Intake Manifold Pressure, bar

291.92 - T_int - Average Intake Manifold Temperature, K

35.388 - v_int - Average Gas Velocity in intake manifold, $\mathrm{m} / \mathrm{s}$ 298.68 - Tw_int - Average Intake Manifold Wall Temperature, K 110.47 - hc_int - Heat Transfer Coeff. in Intake Manifold, W/(m²*K) 183.61 - hc_int.p - Heat Transfer Coeff. in Intake Port, W/(m $\left.{ }^{2 *} \mathrm{~K}\right)$ 73.157 - v_int.p - Max Velocity in a Middle Section of Int. Port, $\mathrm{m} / \mathrm{s}$ 10.035 - A_v.thrt - Total Effective Valve Port Throat Area, $\mathrm{cm}^{2}$ ValveDim. Estim: Num=1 Dv=51.6Dt=44.8Ds=13.4Lv= 8.1 Lv_max=12.9 mm

\section{EXHAUST SYSTEM}

1.0404 - p_exh - Average Exhaust Manifold Gas Pressure, bar 760.12 - T_exh - Average Exhaust Manifold Gas Temperature, K 88.626 - v_exh - Average Gas Velocity in exhaust manifold, $\mathrm{m} / \mathrm{s}$ 16.398 - Sh - Strouhal number: $\mathrm{Sh}=\mathrm{a}^{*} \mathrm{Tau} / \mathrm{L}$ (has to be: $\mathrm{Sh}>8$ ) 697.40 - Tw_exh - Average Exhaust Manifold Wall Temperature, K 216.77-hc_exh-Heat Transfer Coeff. in ExhaustManifold, W/( $\left.\mathrm{m}^{2 *} \mathrm{~K}\right)$ 555.11 - hc_exh.p - Heat Transfer Coeff. in Exhaust Port, W/( $\left.\mathrm{m}^{2 * \mathrm{~K}}\right)$ 236.56 - v_exh.p - Max Velocity in a Middle Section of Exh. Port, m/s 10.472 - A_v.thrt - Total Effective Valve Port Throat Area, $\mathrm{cm}^{2}$ Valve Dim. Estim.: Num=1 Dv=51.2Dt=45.6Ds=13.3 Lv= 6.7Lv_max= $12.8 \mathrm{~mm}$ COMBUSTION

1.7500 - A/F_eq - Air Fiel Equival. Ratio (Lambda) in the Cylinder 0.57143 - F/A_eq - Fuel Air Equivalence Ratio in the Cylinder 79.422 - p_max - Maximum Cylinder Pressure, bar

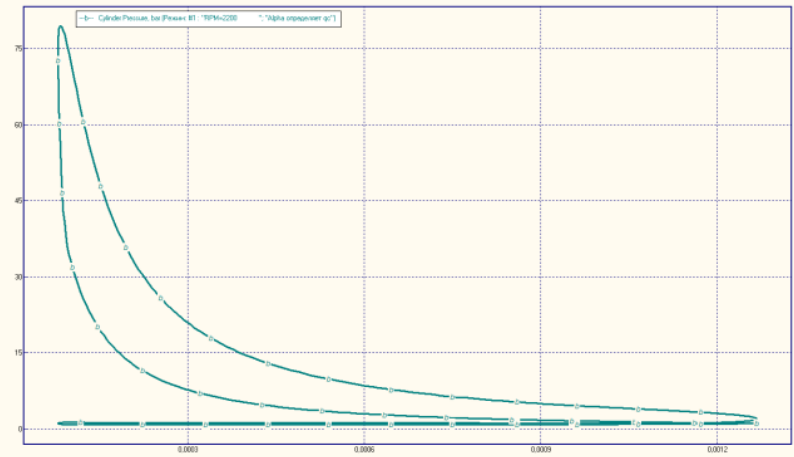

Fig. 2. The schedule of indicator indicators of working process D-240 at work on Biofuel SME B5
1.5000 - Rs_ivc - Swirl Ratio in the Cylinder at IVC 24.870 - W_swirl-Max. Air Swirl Velocity, m/s at cylinder R=33

\section{ECOLOGICAL PARAMETERS}

16.900 - Hartridge- Hartridge Smoke Level

1.7311 - Bosch - Bosch Smoke Number

$0.43585-\mathrm{K}, \mathrm{m}^{-1}$ - Factor of Absolute Light Absorption, 1/m

0.43166 - PM - Specific Particulate Matter emission, g/kWh

$778.32-\mathrm{CO}_{2} \quad$ - Specific Carbon dioxide emission, $\mathrm{g} / \mathrm{kWh}$

1441.2 - NOx.w,ppm- Fraction of wet NOx in exh. gas, ppm

$14.757-\mathrm{NO}_{2}$ - Specif. NOx emis. reduc. to $\mathrm{NO}_{2}, \mathrm{~g} / \mathrm{kWh}$ (Zeldovich)

3.5471 - SE - Summary emission of PM and NOx

$0.01054-\mathrm{SO}_{2} \quad-$ Specific $\mathrm{SO}_{2}$ emission, g/kWh

$\begin{array}{lll}1.2238 & \text { - p_ivc } & \text { - Pressure at IVC, bar } \\ 356.01 & \text { - T_ivc } & \text { - Temperature at IVC, K } \\ 45.504 & \text { - p_tdc } & \text { - Compression Pressure (at TDC), bar } \\ 940.73 & \text { - T_tdc } & \text { - Compression Temperature (at TDC), K } \\ 4.4622 & \text { - p_evo } & \text { - Pressure at EVO, bar } \\ 1086.1 & \text { - T_evo } & \text { - Temperaure at EVO, K } \\ - & -\end{array}$

1121.5 - T_eq - Average Equivalent Temperature of Cycle, $\mathrm{K}$ 343.86 - hc_c - Aver. Factor of Heat Transfer in Cyl., W/m²/K 512.26 - Tw_pist - Average Piston Crown Temperature, K 420.00 - Tw_liner - Average Cylinder Liner Temperature, $\mathrm{K}$ 465.64 - Tw_head - Average Head Wall Temperature, K

381.30 - Tw_cool - Average Temperature of Cooled Surface head of Cylinder Head, $\mathrm{K}$

398.16 - Tboil - Boiling Temp. in Liquid Cooling System, K 10707 - hc_cool - Average Factor of Heat Transfer, W/( $\left.\mathrm{m}^{2 *} \mathrm{~K}\right)$ from head cooled surface to coolant

2143.4 - q_head - Heat Flow in a Cylinder Head, J/s

1991.0 - q_pist - Heat Flow in a Piston Crown, J/s

1480.0 - q_liner - Heat Flow in a Cylinder Liner, J/s

-- MAIN ENGINE CONSTRUCTION PARAMETERS -16.000 - CR - Compression Ratio

4.0000 - n_inj - Number of Injector Nozzles

0.19900 -d_inj - Injector Nozzles Bore, $\mathrm{mm}$

30.000 - Phi_inj - Injection Duration for specif. Inj. Profile, deg.

0.0000 - m_f_ip - Fuel Mass for specified Injection Profile, g

66.000 - EVO - Exhaust Valve Opening, deg. before BDC

16.000 - EVC - Exhaust Valve Closing, deg. after DC

16.000 - IVO - Intake Valve Opening, deg. before DC

46.000 - IVC - Intake Valve Closing, deg. after BDC

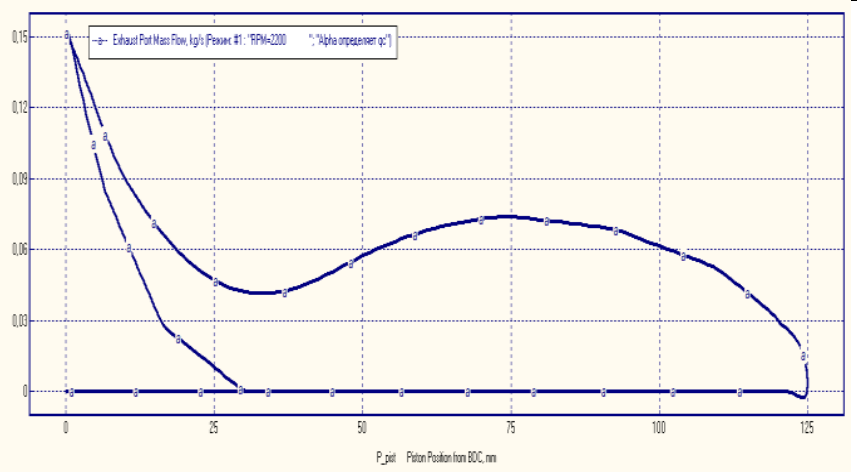

Fig. 3. Environmental performance of D-240 when working on Biofuel SME B5 
Table 2.

Effective performance of D-240 when working on Biofuel SME B20

Fuel: Biofuel SME B20

\section{- PARAMETERS OF EFFICIENCY AND POWER --}

\section{0 - RPM - Engine Speed, rev/min}

57.435 - P_eng - Piston Engine Power, kW

6.5931 - BMEP - Brake Mean Effective Pressure, bar

249.32 - Torque - Brake Torque, $\mathrm{N} \mathrm{m}$

0.05320 - m_f - Mass of Fuel Supplied per cycle, $g$

0.24451 - SFC - Specific Fuel Consumption, $\mathrm{kg} / \mathrm{kWh}$

0.23753 - SFC_ISO - Specific Fuel Consumption in ISO, kg/kWh

0.35753 - Eta_f - Efficiency of piston engine

8.4691 - IMEP - Indicated Mean Effective Pressure, bar

0.45926 - Eta_i - Indicated Efficiency

9.1667 - Sp - Mean Piston Speed, m/s

1.5781 - FMEP - Friction Mean Effective Pressure, bar (Intern.Exp)

0.80687 - Eta_m - Mechanical Efficiency of Piston Engine

\section{ENVIRONMENTAL PARAMETERS}

1.0000 - po_amb - Total Ambient Pressure, bar

288.00 - To_amb - Total Ambient Temperature, $\mathrm{K}$

1.0000 - p_Te - Exhaust Back Pressure, bar (after turbine)

0.98000 - po_afltr - Total Pressure after Induction Air Filter, bar

\section{---- TURBOCHARGING AND GAS EXCHANGE --.-}

0.98000 - p_C - Pressure before Inlet Manifold, bar

288.00 - T_C - Temperature before Inlet Manifold, $\mathrm{K}$

0.09480 - $\mathrm{m} \_$air - Total Mass Airflow (+EGR) of Piston Engine, $\mathrm{kg} / \mathrm{s}$

0.0000 - Eta_TC - Turbocharger Efficiency

1.0557 - po_T - Average Total Turbine Inlet Pressure, bar

776.13 - To_T - Average Total Turbine Inlet Temperature, K

0.09630 - m_gas - Mass Exhaust Gasflow of Pison Engine, kg/s

1.7225 - A/F_eq.t - Total Air Fuel Equivalence Ratio (Lambda)

0.58054 - F/A_eq.t - Total Fuel Air Equivalence Ratio

-0.29788 - PMEP - Pumping Mean Effective Pressure, bar

0.93259 -Eta_v - Volumetric Efficiency

0.91393 -Eta_vo - Volumetric Efficiency defined by Ambient Parameters

0.03556 - x_r $\quad$ - Residual Gas Mass Fraction

0.98454 - Phi - Coeff. of Scavenging (Delivery Ratio / Eta_v) 0.53008 - BF_int-Burnt Gas Fraction Backflowed into the Intake, \%

\subsection{2 - \%Blow-by - \% of Blow-by through piston rings}

\section{INTAKE SYSTEM}

0.97133 - p_int - Average Intake Manifold Pressure, bar

291.94 - T_int - Average Intake Manifold Temperature, $K$

35.392 - v_int - Average Gas Velocity in intake manifold, $\mathrm{m} / \mathrm{s}$

298.69 - Tw_int - Average Intake Manifold Wall Temperature, K 110.47 - hc_int - Heat Transfer Coeff. in Intake Manifold, W/(m²*K) 183.57 - hc_int.p - Heat Transfer Coeff. in Intake Port, $\mathrm{W} /\left(\mathrm{m}^{2 *} \mathrm{~K}\right)$ 73.158 - v_int.p - Max Velocity in a Middle Section of Int. Port, $\mathrm{m} / \mathrm{s}$ 10.035 - A_v.thrt - Total Effective Valve Port Throat Area, $\mathrm{cm}^{2}$ ValveDim. Essim: Num=1 Dv=51.6Dt=44.8Ds=13.4Lv= $8.1 \mathrm{Lv} \_$max $=12.9 \mathrm{~mm}$

\section{EXHAUST SYSTEM}

1.0404 - p_exh - Average Exhaust Manifold Gas Pressure, bar 73.32 - T_exh - Average Exhaust Manifold Gas Temperature, K 89.091 - v_exh - Average Gas Velocity in exhaust manifold, $\mathrm{m} / \mathrm{s}$ 16.540 - Sh - Strouhal number: $\mathrm{Sh}=\mathrm{a} * \mathrm{Tau} / \mathrm{L}$ (has to be: $\mathrm{Sh}>8$ ) 707.74 - Tw_exh - Average Exhaust Manifold Wall Temperature, K 218.15 - hc_exh-Heat TransferCoeff. in Exhaust Manifold, W/( $\left.\mathrm{m}^{2 *} \mathrm{~K}\right)$ 558.66 - hc_exh.p - Heat Transfer Coeff. in Exhaust Port, W/(m²*K)

238.69 - v_exh.p - Max Velocity in a Middle Section of Exh. Port, m/s
1869.1 - T_max - Maximum Cylinder Temperature, $\mathrm{K}$ 6.0000-CA_p.max - Angle of Max. Cylinder Pressure, deg. A.TDC 19.000-CA_tmax -Angle of Max. CylinderTemperature, deg. A.TDC 3.2342 - dp/dTheta- Max. Rate of Pressure Rise, bar/deg. 1.7901 - Ring_Intn- Ringing / Knock Intensity, MW/m2 7665.8 - F_max - Max. Gas Force acting on the piston, $\mathrm{kg}$ System: Custom Fuel Injection System

715.97 - p_inj.max-Max. Sac Injection Pres. (before nozzles), bar 502.84 - p_inj.avr- Mean Sac Press. for Total Fuel Portion, bar 18.467 - d_32 - Sauter Mean Diameter of Drops, microns 20.000 - SOI - Start Of Injection or Ignition Timing, deg. B.TDC 30.136 - Phi_inj - Duration of Injection, CA deg.

12.282 - Phi_ign - Ignition Delay Period, deg.

- ... - calculated by modified Tolstov method : 12.3

7.7178 - SOC - Start of Combustion, deg. B.TDC

0.18651 - x_e.id - Fuel Mass Fraction Evaporated during Ignit. Delay 90.400 - Phi_z - Combustion duration, deg.

Phi_z 5\%= 3.4; Phi_z 50\%= 17.6; Phi_z 95\%= 48.0 3.3561 - Rs_tdc - Swirl Ratio in the Combustion Chamber at TDC 1.5000 - Rs_ivc - Swirl Ratio in the Cylinder at IVC 24.880 - W_swirl -Max. Air Swirl Velocity, m/s at cylinder R= 33

\section{ECOLOGICAL PARAMETERS}

17.133 - Hartridge- Hartridge Smoke Level

1.7516 - Bosch - Bosch Smoke Number

$0.44245-\mathrm{K}, \mathrm{m}^{-1}$ - Factor of Absolute Light Absorption, 1/m 0.43072 - PM - Specific Particulate Matter emission, g/kWh $769.40-\mathrm{CO}_{2}$ - Specific Carbon dioxide emission, $\mathrm{g} / \mathrm{kWh}$ 1289.5 - NOx.w,ppm- Fraction of wet NOx in exh. gas, ppm 13.087 - $\mathrm{NO}_{2}$ - Specif. NOx emis. reduc. to $\mathrm{NO}_{2}, \mathrm{~g} / \mathrm{kWh}$ (Zeldovich) 3.3053 - SE - Summary emission of PM and NOx $0.00513-\mathrm{SO}_{2}-$ Specific $\mathrm{SO}_{2}$ emission, $\mathrm{g} / \mathrm{kWh}$

\section{CYLINDER PARAMETERS}

1.2237 - p_ivc - Pressure at IVC, bar

356.14 - T_ivc - Temperature at IVC, K

45.498 - p_tdc -Compression Pressure (at TDC), bar

940.99 - T_tdc - Compression Temperature (at TDC), K

4.4978 - p_evo - Pressure at EVO, bar

1105.8 - T_evo - Temperaure at EVO, K

\section{---- HEAT EXCHANGE IN THE CYLINDER --.--}

1125.9 - T_eq - Average Equivalent Temperature of Cycle, K 341.49 - hc_c - Aver. Factor of Heat Transfer in Cyl., W/m²/K 512.19 - Tw_pist - Average Piston Crown Temperature, K 420.00 - Tw_liner - Average Cylinder Liner Temperature, K 465.63 - Tw_head - Average Head Wall Temperature, K 381.28 - Tw_cool - Average Temperature of Cooled Surface head of Cylinder Head, K

398.16 - Tboil - Boiling Temp. in Liquid Cooling System, K 10702 - hc_cool - Average Factor of Heat Transfer, W/(m $\left.{ }^{2 *} \mathrm{~K}\right)$ from head cooled surface to coolant

2142.9 - q_head - Heat Flow in a Cylinder Head, J/s

1991.7 - q_pist - Heat Flow in a Piston Crown, J/s

1515.2 - q_liner - Heat Flow in a Cylinder Liner, J/s

-- MAIN ENGINE CONSTRUCTION PARAMETERS -16.000 - CR - Compression Ratio

4.0000 - n_inj - Number of Injector Nozzles 
10.472 - A_v.thrt - Total Effective Valve Port Throat Area, $\mathrm{cm}^{2}$ Valve Dim. Estim: Num=1 Dv=51.2 Dt=45.6Ds=13.3 Lv= 6.7Lv_max $=12.8 \mathrm{~mm}$ COMBUSTION

1.7500 - A/F_eq - Air Fiel Equival. Ratio (Lambda) in the Cylinder 0.57143 - F/A_eq - Fuel Air Equivalence Ratio in the Cylinder 79.629 - p_max - Maximum Cylinder Pressure, bar

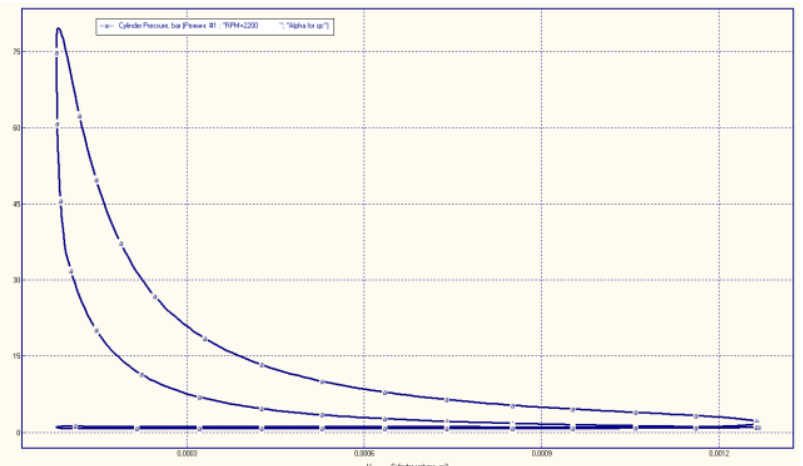

Fig. 4. The schedule of indicator indicators of working process D-240 at work on Biofuel SME B20
0.19900 - d_inj - Injector Nozzles Bore, mm

30.000 - Phi_inj - Injection Duration for specif. Inj. Profile, deg. 0.0000 - m_f_ip - Fuel Mass for specified Injection Profile, $g$ 66.000 - EVO - Exhaust Valve Opening, deg. before BDC 16.000 - EVC - Exhaust Valve Closing, deg. after DC 16.000 - IVO - Intake Valve Opening, deg. before DC 46.000 - IVC - Intake Valve Closing, deg. after BDC

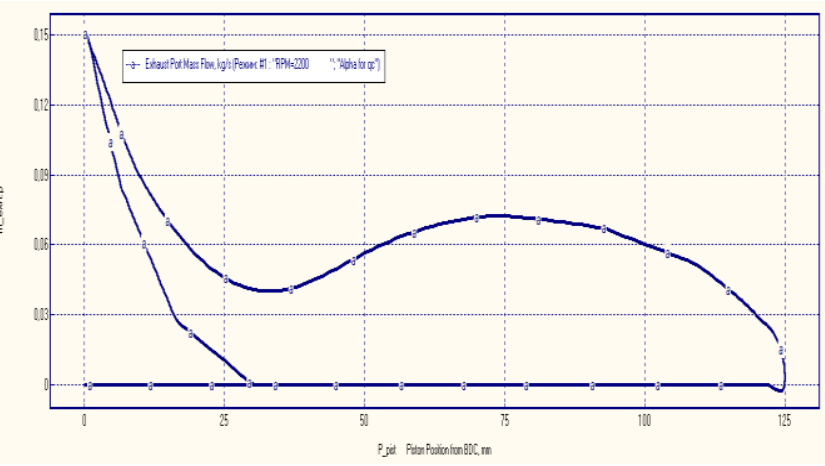

Fig. 5. Environmental performance of the D-240 workflow when working on Biofuel SME B20

\section{Effective performance of D-240 when working on Diesel No. 2}

Table 3.

\section{Fuel: $\quad$ Diesel No. 2}

--- PARAMETERS OF EFFICIENCY AND POWER ---

2200.0 - RPM - Engine Speed, rev/min

58.336 - P_eng - Piston Engine Power, kW

6.6965 - BMEP - Brake Mean Effective Pressure, bar

253.23 - Torque - Brake Torque, $\mathrm{N} \mathrm{m}$

0.05178 - m_f - Mass of Fuel Supplied per cycle, g

0.23433 - SFC - Specific Fuel Consumption, kg/kWh

0.23493 - SFC_ISO - Specific Fuel Consumption in ISO, kg/kWh

0.36148 - Eta_f - Efficiency of piston engine

8.5729 - IMEP - Indicated Mean Effective Pressure, bar

0.46277 - Eta_i - Indicated Efficiency

9.1667 - Sp - Mean Piston Speed, m/s

1.5783 - FMEP - Friction Mean Effective Pressure, bar (Intern.Exp)

0.80926 - Eta_m - Mechanical Efficiency of Piston Engine

\section{----- ENVIRONMENTAL PARAMETERS}

1.0000 - po_amb - Total Ambient Pressure, bar

288.00 - To_amb - Total Ambient Temperature, K

1.0000 - p_Te - Exhaust Back Pressure, bar (after turbine)

0.98000 - po_afltr - Total Pressure after Induction Air Filter, bar

\section{---- TURBOCHARGING AND GAS EXCHANGE -.-.}

0.98000 - p_C - Pressure before Inlet Manifold, bar 288.00 - T_C - Temperature before Inlet Manifold, K 0.09479 - m_air - Total Mass Airflow (+EGR) of Piston Engine, kg/s 0.0000 - Eta_TC - Turbocharger Efficiency

1.0553 - po_T - Average Total Turbine Inlet Pressure, bar 774.28 - To_T - Average Total Turbine Inlet Temperature, K 0.09644 - m_gas - Mass Exhaust Gasflow of Pison Engine, kg/s 1.7224 - A/F_eq.t - Total Air Fuel Equivalence Ratio (Lambda) 0.58057 - F/A_eq.t - Total Fuel Air Equivalence Ratio -0.29806 - PMEP - Pumping Mean Effective Pressure, bar 0.93254 - Eta_v - Volumetric Efficiency 0.91389-Eta_vo-Volumetric Efficiency defined by Ambient Parameters
1889.7 - T_max - Maximum Cylinder Temperature, $\mathrm{K}$ 5.0000-CA_p.max - Angle of Max. CylinderPressure, deg. A.TDC 20.000-CA_tmax -Angle of Max. CylinderTemperature, deg. A.TDC 3.3297 - dp/dTheta- Max. Rate of Pressure Rise, bar/deg. 1.8742 - Ring_Intn- Ringing / Knock Intensity, MW/m² 7802.9 - F_max - Max. Gas Force acting on the piston, $\mathrm{kg}$ System: Custom Fuel Injection System 702.62 - p_inj.max- Max. Sac Injection Pres. (before nozzles), bar 492.07 - p_inj.avr- Mean Sac Press. for Total Fuel Portion, bar 17.842 - d_32 - Sauter Mean Diameter of Drops, microns 20.000 - SOI - Start Of Injection or Ignition Timing, deg. B.TDC

29.813 - Phi_inj - Duration of Injection, CA deg.

13.882 - Phi_ign - Ignition Delay Period, deg.

- ... - calculated by modified Tolstov method : 13.9

6.1176 - SOC - Start of Combustion, deg. B.TDC 0.26612 - x_e.id - Fuel Mass Fraction Evaporated during Ignit. Delay 83.400 - Phi_z - Combustion duration, deg.

Phi_z 5\%= 3.2; Phi_z 50\%= 16.0; Phi_z 95\%= 44.2 3.3558 - Rs_tdc - Swirl Ratio in the Combustion Chamber at TDC 1.5000 - Rs_ivc - Swirl Ratio in the Cylinder at IVC 24.879-W_swirl - Max. Air Swirl Velocity, m/s at cylinder R=33

\section{ECOLOGICAL PARAMETERS}

16.469 - Hartridge- Hartridge Smoke Level

1.6933 - Bosch - Bosch Smoke Number

$0.42366-\mathrm{K}, \mathrm{m}^{-1}$ - Factor of Absolute Light Absorption, 1/m 0.40604 - PM - Specific Particulate Matter emission, g/kWh $755.06-\mathrm{CO}_{2}$ - Specific Carbon dioxide emission, g/kWh 1203.0 - NOx.w,ppm- Fraction of wet NOx in exh. gas, ppm 12.019- $\mathrm{NO}_{2}$ - Specif. NOx emis. reduc. to $\mathrm{NO}_{2}, \mathrm{~g} / \mathrm{kWh}$ (Zeldovich) 3.0705 - SE - Summary emission of PM and NOx $0.00937-\mathrm{SO}_{2} \quad-$ Specific $\mathrm{SO}_{2}$ emission, $\mathrm{g} / \mathrm{kWh}$

\section{------ CYLINDER PARAMETERS}

1.2238 - p_ivc - Pressure at IVC, bar 
Vol. 115, No 4 / 2021

0.03562 - x_r - Residual Gas Mass Fraction

0.98448 - Phi - Coeff. of Scavenging (Delivery Ratio / Eta_v)

0.53147 -BF_int - Burnt Gas Fraction Backflowed into the Intake, \%

$1.5619-\%$ Blow-by - \% of Blow-by through piston rings INTAKE SYSTEM

0.97134 - p_int - Average Intake Manifold Pressure, bar 291.94 - T_int - Average Intake Manifold Temperature, K 35.384 - v_int - Average Gas Velocity in intake manifold, $\mathrm{m} / \mathrm{s}$ 298.69 - Tw_int - Average Intake Manifold Wall Temperature, K 110.46 - hc_int - Heat Transfer Coeff. in Intake Manifold, W/( $\left.\mathrm{m}^{2 *} \mathrm{~K}\right)$ 183.56 - hc_int.p - Heat Transfer Coeff. in Intake Port, W/(m²*K) 73.152 - v_int.p - Max Velocity in a Middle Section of Int. Port, m/s 10.035 - A_v.thrt - Total Effective Valve Port Throat Area, $\mathrm{cm}^{2}$ ValveDim. Estim: Num=1 Dv=51.6Dt=44.8Ds=13.4 Lv= 8.1Lv_max $=12.9 \mathrm{~mm}$ EXHAUST SYSTEM -

1.0404 - p_exh - Average Exhaust Manifold Gas Pressure, bar 771.48 - T_exh - Average Exhaust Manifold Gas Temperature, K 89.011 - v_exh - Average Gas Velocity in exhaust manifold, m/s 16.520 - Sh - Strouhal number: $\mathrm{Sh}=\mathrm{a} * \mathrm{Tau} / \mathrm{L}$ (has to be: $\mathrm{Sh}>8$ ) 706.11 - Tw_exh - Average Exhaust Manifold Wall Temperature, K 217.73 - hc_exh-Heat Transfer Coeff. in Exhaust Manifold, W/(m² $\mathrm{K})$ 557.59 - hc exh.p - Heat Transfer Coeff. in Exhaust Port, W/(m²*K) 238.34 - v_exh.p - Max Velocity in a Middle Section of Exh. Port, m/s 10.472 - A_v.thrt - Total Effective Valve Port Throat Area, $\mathrm{cm}^{2}$ Valve Dim. Estim: $\mathrm{Num}=1 \mathrm{Dv}=51.2 \mathrm{D}=45.6 \mathrm{Ds}=13.3 \mathrm{Lv}=6.7 \mathrm{Lv} \_\mathrm{max}=12.8 \mathrm{~mm}$ COMBUSTION

1.7500 - A/F_eq - Air Fiel Equival. Ratio (Lambda) in the Cylinder 0.57143 - F/A_eq - Fuel Air Equivalence Ratio in the Cylinder 81.054 - p_max - Maximum Cylinder Pressure, bar
356.16 - T_ivc - Temperature at IVC, $\mathrm{K}$
45.501 - p_tdc - Compression Pressure (at TDC), bar
941.10 - T_tdc - Compression Temperature (at TDC), K
4.4915 - p_evo - Pressure at EVO, bar
1102.6 - T_evo - Temperaure at EVO, K

\section{----- HEAT EXCHANGE IN THE CYLINDER}

1129.6 - T_eq - Average Equivalent Temperature of Cycle, K 341.58 - hc_c - Aver. Factor of Heat Transfer in Cyl., W/m²/K 512.87 - Tw_pist - Average Piston Crown Temperature, K 420.00 - Tw_liner - Average Cylinder Liner Temperature, $\mathrm{K}$ 466.12 - Tw_head - Average Head Wall Temperature, K 381.36 - Tw_cool - Average Temperature of Cooled Surface head of Cylinder Head, $\mathrm{K}$

398.16 - Tboil - Boiling Temp. in Liquid Cooling System, K 10722 - hc_cool - Average Factor of Heat Transfer, W/( $\left.\mathrm{m}^{2 *} \mathrm{~K}\right)$

from head cooled surface to coolant

2153.8 - q_head - Heat Flow in a Cylinder Head, J/s

2002.0 - q_pist - Heat Flow in a Piston Crown, J/s

1517.3 - q_liner - Heat Flow in a Cylinder Liner, J/s

--MAIN ENGINE CONSTRUCTION PARAMETERS--

16.000 - CR - Compression Ratio

4.0000 - n_inj - Number of Injector Nozzles

0.19900 - d_inj - Injector Nozzles Bore, mm

30.000 - Phi_inj - Injection Duration for specif. Inj. Profile, deg. 0.0000 - m_f_ip - Fuel Mass for specified Injection Profile, $g$ 66.000 - EVO - Exhaust Valve Opening, deg. before BDC 16.000 - EVC - Exhaust Valve Closing, deg. after DC 16.000 - IVO - Intake Valve Opening, deg. before DC 46.000 - IVC - Intake Valve Closing, deg. after BDC

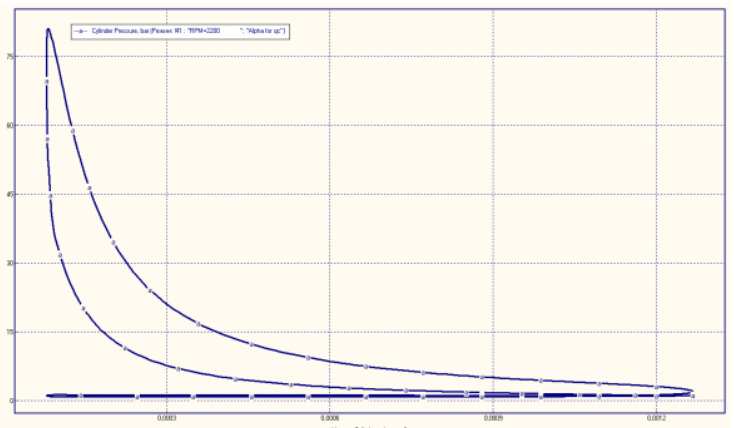

Fig. 6. The schedule of indicator indicators of working process D-240 at work on Diesel No. 2

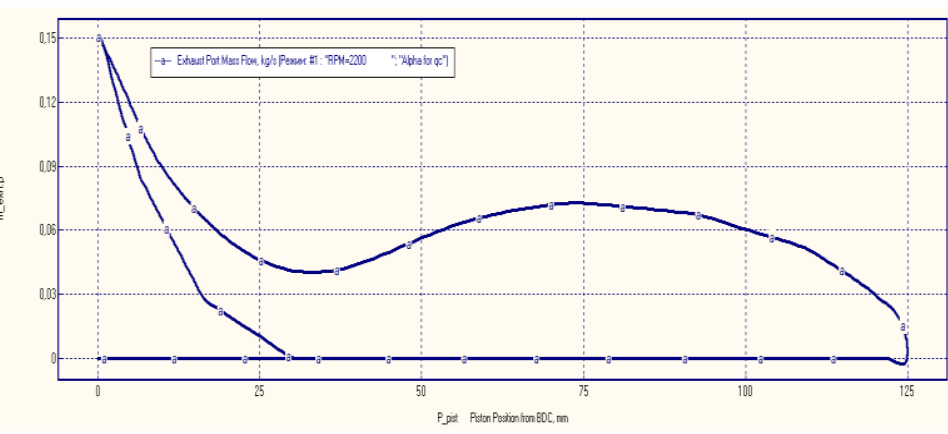

Fig. 7. Environmental performance of the workflow D-240 when working on Diesel No. 2

Table 4.

Effective performance of D-240 when working on Biofuel SME B100

\begin{tabular}{|c|c|}
\hline \multirow{2}{*}{\multicolumn{2}{|c|}{$\begin{array}{c}\text { Fuel: } \quad \text { Biofuel SME B100 } \\
\text {--- PARAMETERS OF EFFICIENCY AND POWER --- }\end{array}$}} \\
\hline & \\
\hline 2200.0 - RPM & - Engine Speed, rev/min \\
\hline 43.804 - P_eng & - Piston Engine Power, kW \\
\hline $5.0284-\mathrm{BMEP}$ & - Brake Mean Effective Press \\
\hline 190.15 - Torque & $-\mathrm{Br}$ \\
\hline $0.05964-\mathrm{m} \_f$ & upplied pe \\
\hline $0.35941-\mathrm{SF} \bar{C}$ & - Specific Fuel Consumption, $\mathrm{kg} / \mathrm{kW}$ \\
\hline 0.30718 -SFC_IS & cific Fuel Consumption in ISO, kg/kWh \\
\hline 0.27654 - Eta_f & - Effi \\
\hline 6.8487 - IMEP & ean Effective Pre \\
\hline 0.37665 - Eta_i & ated Efficiency \\
\hline $9.1667-\mathrm{Sp}$ & Piston Speed, m \\
\hline
\end{tabular}

1463.1 - T_max - Maximum Cylinder Temperature, $\mathrm{K}$ 6.0000 -CA_p.max - Angle of Max. Cylinder Pressure, deg. A.TDC 24.000-CA_tmax - Angle of Max. CylinderTemperature, deg. A.TDC 1.7904 - dp/dTheta- Max. Rate of Pressure Rise, bar/deg. 0.62077 - Ring_Intn- Ringing / Knock Intensity, MW/m2 5993.3 - F_max - Max. Gas Force acting on the piston, $\mathrm{kg}$ System: Custom Fuel Injection System

781.27 - p_inj.max-Max. Sac Injection Pres. (before nozzles), bar 544.44 - p_inj.avr- Mean Sac Press. for Total Fuel Portion, bar 20.367 - d_32 - Sauter Mean Diameter of Drops, microns 20.000 - SOI - Start Of Injection or Ignition Timing, deg. B.TDC 31.605 - Phi_inj - Duration of Injection, CA deg. 3.8835 - Phi_ign - Ignition Delay Period, deg. 
Vol. 115, No 4 / 2021

1.5221 - FMEP - Friction Mean Effective Pressure, bar (Intern.Exp) 0.76764 - Eta_m - Mechanical Efficiency of Piston Engine ENVIRONMENTAL PARAMETERS

1.0000 - po_amb - Total Ambient Pressure, bar 288.00 - To_amb - Total Ambient Temperature, K 1.0000 - p_Te - Exhaust Back Pressure, bar (after turbine) 0.98000 - po_afltr - Total Pressure after Induction Air Filter, bar

TURBOCHARGING AND GAS EXCHANGE -----

0.98000 - p_C - Pressure before Inlet Manifold, bar

288.00 - T_C - Temperature before Inlet Manifold, K 0.09540 - m_air - Total Mass Airflow (+EGR) of Piston Engine, kg/s 0.0000 - Eta_TC - Turbocharger Efficiency

1.0564 - po_T - Average Total Turbine Inlet Pressure, bar 839.10 - To_T - Average Total Turbine Inlet Temperature, $\mathrm{K}$ 0.09606 - m_gas - Mass Exhaust Gasflow of Pison Engine, $\mathrm{kg} / \mathrm{s}$ 1.7228 - A/F_eq.t - Total Air Fuel Equivalence Ratio (Lambda) 0.58044 - F/A_eq.t - Total Fuel Air Equivalence Ratio

-0.29821 - PMEP - Pumping Mean Effective Pressure, bar 0.93858 - Eta v - Volumetric Efficiency

0.91981 -Eta_vo-Volumetric Efficiency defined by Ambient Parameters 0.03374 - x_r - Residual Gas Mass Fraction

$0.98440-\mathrm{Phi}$ - Coeff. of Scavenging (Delivery Ratio / Eta_v) 0.49142 - BF_int - Bumt Gas Fraction Backflowed into the Intake, \% 1.4722 - \% Blow-by - \% of Blow-by through piston rings

\section{INTAKE SYSTEM}

0.97118 - p_int - Average Intake Manifold Pressure, bar 291.93 - T_int - Average Intake Manifold Temperature, K 35.664 - v_int - Average Gas Velocity in intake manifold, $\mathrm{m} / \mathrm{s}$ 298.71 - Tw_int - Average Intake Ma'nifold Wall Temperature, K 110.91 - hc_int - Heat Transfer Coeff. in Intake Manifold, W/( $\left.\mathrm{m}^{2 *} \mathrm{~K}\right)$ 184.41 - hc_int.p - Heat Transfer Coeff. in Intake Port, W/( $\left.\mathrm{m}^{2 *} \mathrm{~K}\right)$ 73.448 - v_int.p - Max Velocity in a Middle Section of Int. Port, m/s 10.035 - A v.thrt - Total Effective Valve Port Throat Area, cm2 ValveDim. Estim: $\mathrm{Num}=1 \mathrm{Dv}=51.6 \mathrm{Dt}=44.8 \mathrm{Ds}=13.4 \mathrm{Lv}=8.1 \mathrm{Lv} \_\max =12.9 \mathrm{~mm}$

\section{EXHAUST SYSTEM}

1.0399 - p_exh - Average Exhaust Manifold Gas Pressure, bar 835.80 - T_exh - Average Exhaust Manifold Gas Temperature, K 95.700 - v_exh - Average Gas Velocity in exhaust manifold, $\mathrm{m} / \mathrm{s}$ 17.195 - Sh - Strouhal number: $\mathrm{Sh}=\mathrm{a}^{*} \mathrm{Tau} / \mathrm{L}$ (has to be: $\mathrm{Sh}>8$ ) 767.69 - Tw_exh - Average Exhaust Manifold Wall Temperature, K 226.27 - hc exh - Heat TransferCoeff. in Exhaust Manifold, W/( $\left.\mathrm{m}^{2 *} \mathrm{~K}\right)$ 579.44 - hc_exh.p - Heat Transfer Coeff. in Exhaust Port, W/(m²*K) 251.43 - v_exh.p - Max Velocity in a Middle Section of Exh. Port, m/s 10.472 - A_v.thrt - Total Effective Valve Port Throat Area, $\mathrm{cm}^{2}$ ValveDim. Estim: $\mathrm{Num}=1 \mathrm{Dv}=51.2 \mathrm{D}=45.6 \mathrm{Ds}=13.3 \mathrm{Lv}=6.7 \mathrm{Lv} \_\mathrm{max}=12.8 \mathrm{~mm}$ COMBUSTION

1.7500 - A/F_eq - Air Fiel Equival. Ratio (Lambda) in the Cylinder 0.57143 - F/A_eq - Fuel Air Equivalence Ratio in the Cylinder 62.256 - p_max - Maximum Cylinder Pressure, bar
- ... - calculated by modified Tolstov method : 3.9 16.117 - SOC - Start of Combustion, deg. B.TDC 0.00427 - x_e.id - Fuel Mass Fraction Evaporated during Ignit. Delay 229.40 - Phi_z - Combustion duration, deg.

Phi_z 5\%=6.8; Phi_z 50\%= 36.4; Phi_z 95\%=141.4 3.3619-Rs_tdc-Swirl Ratio in the Combustion Chamber at TDC 1.5000 - Rs_ivc - Swirl Ratio in the Cylinder at IVC 24.924 - W_swirl - Max. Air Swirl Velocity, m/s at cylinder R=33 --:-- ECOLOGICAL PARAMETERS

60.291 - Hartridge- Hartridge Smoke Level 4.4388 - Bosch - Bosch Smoke Number $2.1485-\mathrm{K}, \mathrm{m}^{-1}$ - Factor of Absolute Light Absorption, 1/m 2.1593 - PM - Specific Particulate Matter emission, g/kWh 1029.1 - $\mathrm{CO}_{2}$ - Specific Carbon dioxide emission, $\mathrm{g} / \mathrm{kWh}$ 663.50 - NOx.w,ppm- Fraction of wet NOx in exh. gas, ppm $8.8930-\mathrm{NO}_{2}$ - Specif. $\mathrm{NOx}$ emis. reduc. to $\mathrm{NO}_{2}, \mathrm{~g} / \mathrm{kWh}$ (Zeldovich) 8.4681 - $\mathrm{SE}$ - Summary emission of PM and NOx $0.03594-\mathrm{SO}_{2} \quad-$ Specific $\mathrm{SO}_{2}$ emission, $\mathrm{g} / \mathrm{kWh}$

\section{CYLINDER PARAMETERS}

1.2229 - p_ivc - Pressure at IVC, bar

354.45 - T_ivc - Temperature at IVC, K

45.399 - p_tdc - Compression Pressure (at TDC), bar 935.15 - T_tdc - Compression Temperature (at TDC), K 4.8959 - p_evo - Pressure at EVO, bar 1209.1 - T_evo - Temperaure at EVO, K HEAT EXCHANGE IN THE CYLINDER

960.27 - T_eq - Average Equivalent Temperature of Cycle, K 306.33 - hc_c - Aver. Factor of Heat Transfer in Cyl., W/m²/K 480.69 - Tw_pist - Average Piston Crown Temperature, K 20.00 - Tw_liner - Average Cylinder Liner Temperature, K 437.00 - Tw__head - Average Head Wall Temperature, K 377.11 - Tw_cool - Average Temperature of Cooled Surface head of Cylinder Head, $\mathrm{K}$

398.16 - Tboil - Boiling Temp. in Liquid Cooling System, K 9491.6 - hc_cool - Average Factor of Heat Transfer, W/( $\left.\mathrm{m}^{2 *} \mathrm{~K}\right)$ from head cooled surface to coolant

1523.3 - q_head - Heat Flow in a Cylinder Head, J/s 1396.1 - q_pist - Heat Flow in a Piston Crown, J/s 1535.3 - q_liner - Heat Flow in a Cylinder Liner, J/s --MAIN ENGINE CONSTRUCTION PARAMETERS-16.000 - CR - Compression Ratio

4.0000 - n inj - Number of Injector Nozzles 0.19900 - $\mathrm{d}$ _inj - Injector Nozzles Bore, mm 30.000 - Phi_inj - Injection Duration for specif. Inj. Profile, deg. 0.0000 - m_f ip - Fuel Mass for specified Injection Profile, $g$ 66.000 - EVO - Exhaust Valve Opening, deg. before BDC 16.000 - EVC - Exhaust Valve Closing, deg. after DC 16.000 - IVO - Intake Valve Opening, deg. before DC 46.000 - IVC - Intake Valve Closing, deg. after BDC

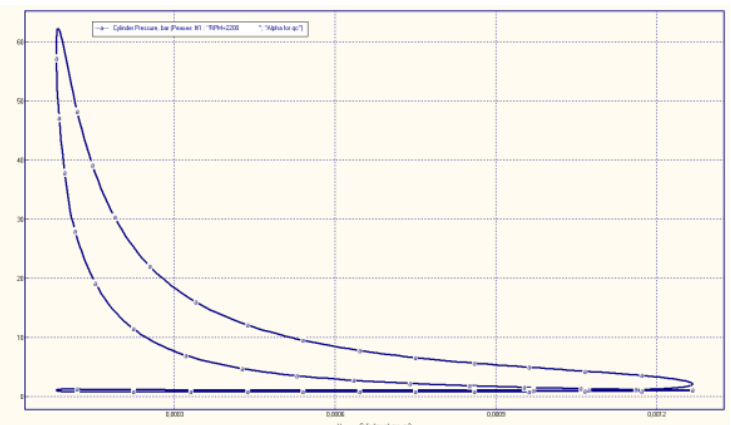

Fig. 8. The schedule of indicator indicators of working process D-240 when working on Biofuel SME B100

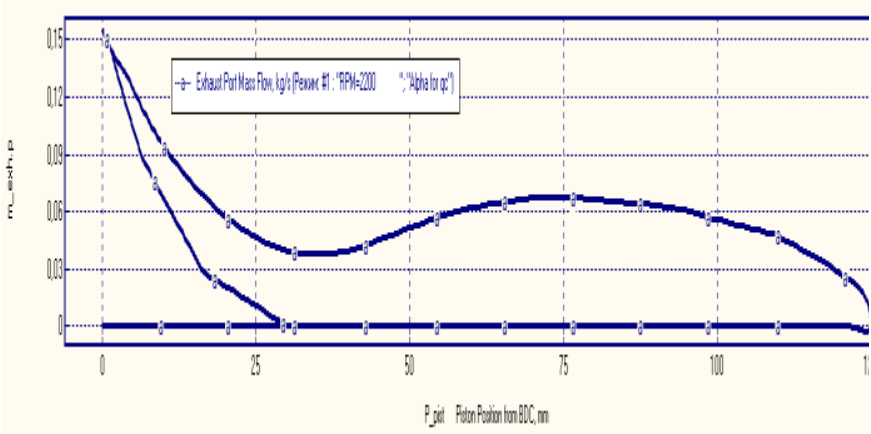

Fig. 9. Environmental performance of D-240 when working on Biofuel SME B100 


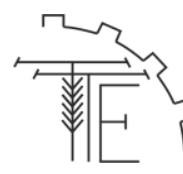

The shortest way to the widespread use of liquid biofuels as fuel in tractor engines without changing their design is to improve their fuel systems through the introduction and use of new processes in the technological cycle of fuel preparation. use of two-fuel systems, including systems of supply of oil and biofuels, use of regular oil fuel as starting and reserve fuel

As for the economic indicators of work, according to preliminary estimates (excluding the cost of restoring soil fertility), the benefits of using biofuels are obvious.

Environmental indicators that determine the safety of human life and associated with the use of agricultural machinery that consumes biofuels by $8-10 \%$ are better than the use of mineral diesel fuel.

\section{Conclusions}

1. Because biofuels contain oxygen-containing substances $(O=10.9 \%)$, its heat of combustion is lower $\left(Q_{n}=39.45 \mathrm{MJ} / \mathrm{kg}\right)$ than that of diesel fuel $\left(Q_{n}=42\right), 5 \mathrm{MJ} / \mathrm{kg}$, Figure 13) $(O=0.4 \%)$. This fact leads to a decrease in diesel power (up to $25 \%$ in nominal mode).

2. Compared with diesel fuel, the specific consumption of biofuel in the nominal mode of operation of the engine increased by $37 \%$.

3. Studies of fuel injection and mixing show that the average droplet diameter in the MERO study increased to $20 \%$, which increases the range of the jet compared to diesel fuel and adversely affects the mixing process. And it burns.

4. The use of vegetable alternative fuels with a reduction of carbon content by $10 \%$ can reduce $\mathrm{CO}_{2}$ emissions. The rate of formation of soot particles during the combustion of biofuels is 8.8 times higher than the rate of combustion of diesel fuel.

Recommendations. In order for the technical and economic performance of MERO to reach the optimal level, it is necessary to strengthen such processes as cleaning, injection, mixing and incineration. Heating of the injected fuel $\left(\sim 70{ }^{\circ} \mathrm{C}\right)$ can positively influence these processes, which will lead to the improvement of physicochemical parameters of the fuel, increase of the fuel injection pressure ( $\sim 80 \mathrm{MPa})$ will also reduce the diameter of sprayed fuel droplets, air turbulence. evaporation and stirring.

\section{Список використаних джерел}

1. Eberhart S. A., Russel W. A. Stability parameters for comparing varieties. Crop Sci. 1986. V. 6. № 1. P. 34-40.

2. Калетнік Г. М. Біопалива: ефективність їх виробництва та споживання в АПК України: навч. посібник. К: Аграрна наука, 2010. 327 с.

3. Окоча А. І., Антипенко А. М. Паливно-мастильні та інші експлуатаційні матеріали. К.: Урожай, 1996. $336 \mathrm{c}$.

4. Murugesan A., Subramanian R., Nedunchezhian N. Biodiesel as an alternative fuel for diesel engines. Renew sust energy rev. 2009. P. 653-662.

5. Семенов В. Біодизельне паливо для України. Вісник Національної академії наук України. 2007. № 4. C. 18-22.

6. Tokarchuk D. M., Pryshliak N. V., Tokarchuk O. A., Mazur K. V. Technical and economic aspects of biogas production at a small agricultural enterprise with modeling of the optimal distribution of energy. INMATEH - Agricultural Engineering. 2020. Vol. 61. №. 2. P. 339-349.

7. Анісімов В. Ф., Яцковський В. І., П'ясецький А. А. Рябошапка В. Б. Напрямки створення багатопаливних двигунів на базі дизельного циклу. Промислова гідравліка і пневматика. 2011. №2 (32). C. 100-105.

8. Бурлака С. А., Явдик В. В., Сленич А. П. Методи досліджень та способи оцінки впливу палив 3 відновлюваних ресурсів на роботу дизельного двигуна. Вісник Хмельницького національного університету. 2019. №2 (271). С. 212-220.

9. Малаков О. І., Бурлака С. А., Михальова Ю. О. Математичне моделювання та основи конструювання вібраційних змішувачів. Вісник Хмельнищького наџіонального університету. 2019. № 5 (277). С. 30-33. 


\section{Reference}

[1] Eberhart, S. A., Russel, W. A. (1986). Stability parameters for comparing varieties. Crop Sci. 6(1). 3440. [in English].

[2] Kaletnik, H.M. (2010). Biopalyva: efektyvnist' yikh vyrobnytstva ta spozhyvannya v APK Ukrayiny: navch. posibnyk. K: Ahrarna nauka. [In Ukrainian].

[3] Okocha, A. I., Antypenko, A. M. (1996). Palyvno-mastyl'ni ta inshi ekspluatatsiyni materialy. K.: Urozhay. [in Ukrainian].

[4] Murugesan, A., Subramanian, R., Nedunchezhian, N. (2009). Biodiesel as an alternative fuel for diesel engines. Renew sust energy rev. 653-662. [in English].

[5] Semenov, V. (2007). Biodyzel'ne palyvo dlya Ukrayiny. Visnyk Natsional'noyi akademiyi nauk Ukrayiny, 4. 18-22. [in Ukrainian].

[6] Tokarchuk, D. M., Pryshliak, N. V., Tokarchuk, O. A., Mazur, K. V. (2020). Technical and economic aspects of biogas production at a small agricultural enterprise with modeling of the optimal distribution of energy. INMATEH - Agricultural Engineering. 61(2). 339-349. [in English].

[7] Anisimov, V. F., Yatskovs'kyy, V. I., P'yasets'kyy, A. A., Ryaboshapka, V. B. (2011). Napryamky stvorennya bahatopalyvnykh dvyhuniv na bazi dyzel'noho tsyklu. Promyslova hidravlika i pnevmatyka. 2 (32). 100-105. [in Ukrainian].

[8] Burlaka, S.A., Yavdyk, V.V., Yelenych, A.P. (2019). Metody doslidzhen' ta sposoby otsinky vplyvu palyv z vidnovlyuvanykh resursiv na robotu dyzel'noho dvyhuna. Visnyk Khmel'nyts'koho natsional'noho universytetu. 2 (271). 212-220. [in Ukrainian].

[9] Malakov, O.I., Burlaka, S.A., Mykhal'ova, Y.O. (2019). Matematychne modelyuvannya ta osnovy konstruyuvannya vibratsiynykh zmishuvachiv. Visnyk Khmel'nyts'koho natsional'noho universytetu. 5(277). 30-33. [in Ukrainian].

\section{ЗНЯТТЯ ТЕХНІКО-ЕКОНОМІЧНИХ ПОКАЗНИКІВ ДВИГУНА Д-240 ПРИ ВИКОРИСТАННЯ БІОПАЛИВА ШЛЯХОМ ЗАСТОСУВАННЯ ПРОГРАМНОГО КОМПЛЕКСУ ДИЗЕЛЬ-РК}

Украӥна є однією з енергетично дефіцитних краӥн, оскільки покриває свої потреби в паливноенергетичних ресурсах за рахунок внутрішніх запасів лише на 53\% (імпортує 75\% необхідного природного газу та 85\% сирої нафти та нафтопродуктів). Залежність від імпорту нафти більшість розвинених країн розглядає як питання національної та енергетичної безпеки, а використання нафтопродуктів як джерел енергії створює значний екологічний ризик.

Згідно з аналізом стану світової машинобудівної промисловості, найбільш ефективними заходами щьодо вдосконалення конструкиії двигуна є: розробка та впровадження нового робочого прочесу з ефективними методами змішування та згоряння; розробка та створення конструкиіі системи автоматичного регулювання обертання дизеля. частота для покращення економї палива, $a$ для зниження токсичності в режимі часткового навантаження деякі ичліндри вимикаються на холостому ходу.

Для посилення вимог до нормативів витрат палива та рівня токсичності відпрачьованих газів, а також підвищення надійності та ефективності сільськогосподарських силових двигунів необхідно сформулювати заходи щодо вдосконалення його конструкиії.

Вибір палива має визначатися оптимальним поєднанням екологічних та економічних показників двигуна. Відзначаються перспективи використання того чи іншого палива рослинного походження. Якщо для палива нафтового походження поліпшення його властивостей визначається спеціальною обробкою, то олії із заданими характеристиками можна отримати вже в «польові» иляхом підбору відповідних сортів рослин, застосування добрив, агротехнічних заходів тощьо.

Проблему зниження витрати дизельного палива на холостому ходу та малому навантаженні можна вирішити, виключивши з його роботи частину ияліндрів (цей метод широко використовується) $i$ замикаючи иикл одного ииліндра.Проведено порівняльний аналіз основних технічних та екологічних показників дизельного двигуна Д-240 при роботі на традиційному та альтернативному паливі з використанням комп'ютерної програми Дизель-РК.

Ключові слова:. діагностика, двигун, індикатори, біопаливо, Д-240, індикаторні показники, техніко-економічні характеристики

Ф. 2. Рис. 9. Табл. 4. Літ. 9. 


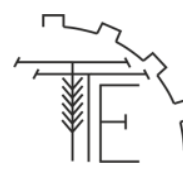

\section{СНЯТИЕ ТЕХНИКО-ЭКОНОМИЧЕСКИХПОКАЗАТЕЛЕЙ ДВИГАТЕЛЯ Д-240 ПРИ ИСПОЛЬЗОВАНИИ БИОТОПЛИВА ПУТЕМ ПРИМЕНЕНИЯ ПРОГРАММНОГО КОМПЛЕКСА ДИЗЕЛЬ-РК}

Украина является одной из энергетически дефицитных стран, поскольку покрывает свои потребности в топливно-энергетических ресурсах за счет внутренних запасов всего на 53\% (импортирует 75\% необходимого природного газа и 85\% сырой нефти и нефтепродуктов). В зависимости от импорта нефти больиинство развитых стран рассматривают как вопросы национальной и энергетической безопасности, а использование нефтепродуктов как источников энергии создает значительный экологический риск.

Согласно анализу состояния мировой машиностроительной промышленности, наиболее эффективными мерами по усовершенствованию конструкиии двигателя являются: разработка и внедрение нового рабочего прочесса с эффективными методами смешивания и сгорания; разработка и создание конструкции системы автоматической регулировки вращения дизеля. частота для улучшения экономии топлива, а для снижения токсичности в частичной нагрузке некоторые цчилиндры выключаются на холостом ходу.

Для усиления требований к нормативам расхода топлива и уровня токсичности отработавиих газов, а также повышения надежности и эффективности сельскохозяйственных силовых двигателей необходимо сформулировать меры по усовершенствованию его конструкции.

Выбор топлива должен определяться оптимальным сочетанием экологических и экономичных показателей двигателя. Отмечаются перспективы использования того или иного топлива растительного происхождения. Если для топлива нефтяного происхождения улучшение его свойств определяется специальной обработкой, то масла с заданными характеристиками можно получить уже в полеве путем подбора соответствующих сортов растений, применения удобрений, агротехнических мероприятий и т.п.

Проблему снижения расхода дизельного топлива на холостом ходу и малой нагрузке можно решить, исключив из его работы часть цилиндров (этот метод иироко используется) и замькая цүикл одного цүилиндра.Проведен сравнительный анализ основных технических и экологических показателей дизельного двигателя Д-240 при работе на традиционном и альтернативном топливе с использованием компьютерной программы Дизель-РК.

Ключевые слова:. диагностика, двигатель, индикаторы, биотопливо, Д-240, индикаторные показатели, технико-экономические характеристики

Ф. 2. Рис. 9. Табл. 4. Лит. 9.

\section{INFORMATION ABOUT THE AUTHORS}

Serhii Burlaka - Doctor of Philosophy, Senior Lecturer, Department of Technological Processes and Equipment of Processing and Food Production, Vinnytsia National Agrarian University (3 Solnechnaya St., Vinnytsia, 21008, Ukraine, e-mail: ipserhiy@gmail.com, https://orcid.org/ 0000-0002-4079-4867).

Oleksii Tokarchuk - Ph.D., Associate Professor of the Department of technological processes and equipment for processing of the Vinnytsia National Agrarian University (3, Sonyachna st., Vinnytsia, Ukraine, 21008, email: tokarchyk08@ net.ukr, https://orcid.org/0000-0001-8036-1743).

Бурлака Сергій Андрійович - доктор філософії, старший викладач кафедри «Технологічних процесів та обладнання переробних і харчових виробництв» Вінницького національного аграрного університету (вул. Сонячна, 3, м. Вінниця, 21008, Україна, e-mail: ipserhiy@ gmail.com, https://orcid.org/ 0000-0002-4079-4867) Токарчук Олексій Анатолійович - кандидат технічних наук, доцент кафедри технологічних процесів та обладнання переробних і харчових виробництв Вінницького національного аграрного університету (вул. Сонячна, 3, м. Вінниця, 21008, Україна, e-mail: tokarchyk08@ukr.net, https://orcid.org/0000-0001-8036-1743).

Бурлака Сергей Андреевич - доктор философии, старший преподаватель кафедры «Технологических процессов и оборудования перерабатывающих и пищевых производств» Винницкого национального аграрного университета (ул. Солнечная, 3, г. Винница, 21008, Украина, e-mail: ipserhiy@ gmail.com, https:/orcid.org/ 0000-0002-4079-4867)

Токарчук Алексей Анатольевич - кандидат технических наук, доцент кафедры технологических процессов и оборудования перерабатывающих и пищевых производств Винницкого национального аграрного университета (ул. Солнечная, 3, г. Винница, 21008, Украина, e-mail: tokarchyk08@ukr.net, https://orcid.org/0000-0001-8036-1743). 